Pacific

Journal of

Mathematics

\title{
ON ROTATIONALLY INVARIANT SHRINKING RICCI SOLITONS
}

BRETT KOTSCHWAR 


\title{
ON ROTATIONALLY INVARIANT SHRINKING RICCI SOLITONS
}

\author{
BRETT KOTSCHWAR
}

\begin{abstract}
We study the gradient Ricci shrinking soliton equation on rotationally symmetric manifolds of dimensions $n \geq 3$ and prove that the only complete metrics on $S^{n}, \mathbb{R}^{n}$, and $\mathbb{R} \times S^{n-1}$ that admit shrinking soliton structures are respectively the round, flat, and standard cylindrical metrics.
\end{abstract}

\section{Introduction}

Recall that a Riemannian manifold $(M, g)$ is said to be a shrinking gradient Ricci soliton provided

$$
\operatorname{Rc}(g)+\nabla \nabla f-\lambda g=0
$$

for some $f \in C^{\infty}(M)$ and $\lambda>0$. The analogous objects in the cases $\lambda=0$ and $\lambda<0$ are known as steady and expanding solitons, respectively, and the triple $(g, f, \lambda)$ is referred to as a (gradient) soliton structure on the manifold $M$. Solitons correspond to self-similar solutions of the Ricci flow and arise commonly in the analysis of its singularities.

As is clear from (1), any Einstein metric gives rise, rather trivially, to at least one soliton structure on a manifold. For non-Einstein examples, it is natural to consider metrics with many symmetries and perhaps first to look among the class of rotationally symmetric metrics. In all dimensions greater than one, this class has been shown to contain complete, nontrivial examples of steady and expanding solitons; see for example [Ivey 1992; Chow et al. 2007]. However, one does not expect to find corresponding examples in the shrinking case, and the purpose of this work is to confirm this expectation. Our main result is a classification theorem:

Theorem 1. Suppose $n \geq 3$, and $(g, f, \lambda)$ is a complete, rotationally invariant shrinking soliton structure on a on a manifold $M^{n}$ diffeomorphic to one of $S^{n}, \mathbb{R}^{n}$, or $\mathbb{R} \times S^{n-1}$. Then

(1) if $M^{n} \cong S^{n}$, then $g$ is isometric to a round sphere and $f \equiv$ const;

(2) if $M^{n} \cong \mathbb{R}^{n}$, then $g$ is flat;

MSC2000: primary 53C44; secondary 58J35.

Keywords: Ricci solitons, Ricci flow. 
(3) if $M^{n} \cong \mathbb{R} \times S^{n-1}$, then $g$ is isometric to the standard cylinder $d r^{2}+\omega_{0}^{2} g_{S^{n-1}}$ of radius $\omega_{0}=\sqrt{(n-2) / \lambda}$ and $f=f(r)=(n-2) r^{2} /\left(2 \omega_{0}^{2}\right)+$ linear.

This result is known (in greater generality) in dimensions less than four. In dimension two, Hamilton $[1995 ; 1988]$ has shown that the only complete metrics that admit shrinking soliton structures are the flat metric on $\mathbb{R}^{2}$ and the round metric on $S^{2}$. In fact, it is not difficult to show that any potential candidate on a surface must be rotationally symmetric; see [Hamilton 1995]. This observation has also been used, for example, by Chen, $\mathrm{Lu}$, and Tian [2006] to provide an alternative proof of the uniqueness of the constant curvature soliton on $S^{2}$. In a sense, then, our result is an extension of these two-dimensional findings to higher dimensions.

In dimensions greater than two, gradient solitons need not be rotationally symmetric; nevertheless, shrinking solitons have been classified in dimension three in [Perelman 2003]. There it is shown that the only complete, nonflat examples of bounded nonnegative sectional curvature are either quotients of the round sphere $S^{3}$ or of the standard cylinder $S^{2} \times \mathbb{R}$. As it follows from an estimate of Hamilton and Ivey [1995; 1992] that three-dimensional ancient solutions of bounded curvature necessarily have nonnegative sectional curvature, Perelman's argument effectively classifies all such potential examples. Perelman has further asserted that the classification stands without the assumption of bounded curvature.

At present, there are no such complete classifications in higher dimensions. Ancient solutions no longer need have nonnegative sectional curvature, and indeed, beginning in dimension four, there are complete examples of non-Einstein shrinking solitons with curvatures of mixed sign; see for example [Koiso 1990]. However, there are some partial results. A consequence of the recent work of Böhm and Wilking [2006], for example, is that the only compact shrinking soliton with 2-positive curvature operator is the round sphere. In the Kähler category, Feldman, Ilmanen, and Knopf [2003] have obtained results similar to our own for $U(n)$ invariant Kähler-Ricci shrinking solitons. In particular, their [Proposition 9.2] that the flat metric is the only complete $U(n)$-invariant gradient shrinking soliton on $\mathbb{C}^{n}$ - is the Kähler analog of the second case of our Theorem 1. Their paper also provides nontrivial examples of $U(n)$-invariant gradient solitons (of all types) on other spaces. Also, $\mathrm{Ni}$ [2005] has shown that any complex $m$-dimensional nonflat gradient shrinking Kähler-Ricci soliton of positive bisectional curvature must be compact and isometric-biholomorphic to $\mathbb{C} P^{m}$ with the Fubini-Study metric. Together with the aforementioned result of Böhm and Wilking, it is thus reasonable to expect that Perelman's three-dimensional result - that there are no complete, noncompact shrinking solitons of strictly positive sectional curvature - may extend to higher dimensions. The results of this paper are further evidence in support of this conjecture. 
To prove Theorem 1, we show first that, under the constraint (1), the rotational symmetry of a nonflat metric implies the rotational symmetry of the gradient function. This reduces the proof of the proposition to the study of a certain second order system of nonlinear ODEs. By a change of variables due to Robert Bryant and Tom Ivey [Bryant $\leq$ 1992; Ivey 1992; Chow et al. 2007], we are able to further reduce the problem to the study of an equivalent first-order system amenable to phaseplane analysis. For the case $M^{n} \cong S^{n}$, we show that any candidate metric must have positive curvature operator and then apply the result of Böhm and Wilking. It is no doubt possible to prove this case solely from the analysis of the system of ODEs (without using any of the dynamic properties of the Ricci flow); however we content ourselves here with a proof by the most readily available means. For the noncompact cases, we use the criterion of completeness to eliminate all but the two standard metrics by their asymptotic behavior.

\section{The soliton condition for rotationally symmetric manifolds.}

2.1. The warped-product metric. Fix $n>1$, and let $\tilde{g}$ denote the metric on $S^{n}$ of constant sectional curvature 1. For $-\infty \leq A<\Omega \leq \infty$ and positive functions $\omega \in C^{\infty}(A, \Omega)$, we shall consider the warped-product metric $g=d r^{2}+\omega^{2}(r) \tilde{g}$ on the cylinder $\mathscr{C}_{A, \Omega}:=(A, \Omega) \times S^{n}$. For this metric, there is a standard lemma:

Lemma 2. The metric $g=d r^{2}+\omega(r)^{2} \tilde{g}$ on $\mathscr{C}_{0, \Omega}$ extends to a smooth metric on $B_{\Omega}(\mathbf{0}) \subset R^{n+1}$ if and only if $\Omega>0$ and

$\lim _{r \rightarrow 0} \omega(r)=0, \quad \lim _{r \rightarrow 0} \omega^{\prime}=1, \quad$ and $\quad \lim _{r \rightarrow 0} \frac{d^{2 k} \omega}{d r^{2 k}}(r)=0 \quad$ for all $k$.

The metric extends to a smooth metric on $S^{n+1}$ if and only if also $\Omega<\infty$ and

$$
\lim _{r \rightarrow \Omega} \omega(r)=0, \quad \lim _{r \rightarrow \Omega} \omega^{\prime}=-1, \quad \text { and } \quad \lim _{r \rightarrow \Omega} \frac{d^{2 k} \omega}{d r^{2 k}}(r)=0 \quad \text { for all } k .
$$

Proof. See for example [Chow and Knopf 2004, Lemma 2.10].

Thus we may accomplish the proof of Theorem 1 by the study of soliton structures on $\mathscr{C}_{A, \Omega}$. We begin by recording the expressions of a few geometric quantities associated to $g$.

The sectional curvatures of $g$ corresponding to two-planes tangent to the radial direction and to two-planes spanned by two orbital directions (that is, by two directions tangent to the spherical factor) are respectively

$$
v_{1}=-\frac{\omega^{\prime \prime}}{\omega} \quad \text { and } \quad v_{2}=\frac{1-\left(\omega^{\prime}\right)^{2}}{\omega^{2}} .
$$


More generally, the action of the operator $\mathrm{Rm}: \Lambda^{2} M^{n+1} \rightarrow \Lambda^{2} M^{n+1}$ for arbitrary $\theta^{1}, \theta^{2} \in \Lambda^{1} S^{n}$ is determined by

$$
\operatorname{Rm}\left(\theta^{1} \wedge d r\right)=v_{1} \theta^{1} \wedge d r \quad \text { and } \quad \operatorname{Rm}\left(\theta^{1} \wedge \theta^{2}\right)=v_{2} \theta^{1} \wedge \theta^{2} .
$$

From this we see that the range $\left[\min \left\{v_{1}, v_{2}\right\}, \max \left\{v_{1}, v_{2}\right\}\right]$ contains all the sectional curvatures of $g$ and that the positivity of the sectional curvature - and also of the curvature operator $\mathrm{Rm}$ - is implied by that of $v_{1}$ and $\nu_{2}$.

Finally, we note that the Ricci curvature of $g$ has the form

$$
\operatorname{Rc}(g)=-n \frac{\omega^{\prime \prime}}{\omega} d r^{2}+\left((n-1)\left(1-\left(\omega^{\prime}\right)^{2}\right)-\omega \omega^{\prime \prime}\right) \tilde{g},
$$

where the prime denotes differentiation with respect to $r$.

2.2. The soliton equation. A routine computation also shows that for a smooth function $f$ on $\mathscr{C}_{A, \Omega}$, one has

$$
\nabla \nabla f=\left\{\begin{array}{l}
\nabla_{0} \nabla_{0} f=f_{00}, \\
\nabla_{0} \nabla_{i} f=f_{i 0}-\left(\omega^{\prime} / \omega\right) f_{i}, \\
\nabla_{i} \nabla_{j} f=\widetilde{\nabla}_{i} \widetilde{\nabla}_{j} f+\omega \omega^{\prime} f_{0} \tilde{g}_{i j}
\end{array}\right.
$$

in local coordinates $\left(\theta^{0}=r, \theta^{i}\right){ }^{1}$

In these coordinates, (1) implies the equations

$$
\begin{aligned}
& 0=f_{00}-n\left(\omega^{\prime \prime} / \omega\right)-\lambda, \\
& 0=f_{0 i}-\left(\omega^{\prime} / \omega\right) f_{i}, \\
& 0=\widetilde{\nabla}_{i} \widetilde{\nabla}_{j} f+\left[(n-1)\left(1-\left(\omega^{\prime}\right)^{2}\right)-\omega \omega^{\prime \prime}+\omega \omega^{\prime} f_{0}-\lambda \omega^{2}\right] \tilde{g}_{i j},
\end{aligned}
$$

which further reduce to the system

$$
\begin{aligned}
f^{\prime \prime}-\lambda & =n \omega^{\prime \prime} / \omega, \\
\omega \omega^{\prime} f^{\prime}-\lambda \omega^{2} & =\omega \omega^{\prime \prime}+(n-1)\left(\left(\omega^{\prime}\right)^{2}-1\right)
\end{aligned}
$$

when $f$ is a radial function.

The equations (4)-(6) involve the partial derivatives of $f$ and, despite the rotational symmetry of the metric and the Ricci curvature, there is no a priori reason to assume that $f$ shares this symmetry. Nevertheless, as we show next, this symmetry is, in fact, implied unless $g$ is flat, and thus for the proof of Theorem 1, there is no loss in restricting our attention to the case $f=f(r)$.

${ }^{1}$ Here and throughout, when working in coordinates $\theta^{0}, \ldots, \theta^{n}$, we shall use Roman letters to denote indices $1, \ldots, n$ and use a tilde to denote quantities (Levi-Civita connection, curvature, etc.) associated to the metric $\tilde{g}$ on $S^{n}$. In particular, $\widetilde{\nabla}_{i} \widetilde{\nabla}_{j} f$ represents the Hessian of $f(r, \cdot)$ considered as a function on $S^{n}$. 
Proposition 3. Suppose that $(g, f, \lambda)$ is a complete, rotationally symmetric gradient shrinking soliton structure on $M^{n+1} \cong S^{n+1}, \mathbb{R}^{n+1}$, or $\mathbb{R} \times S^{n}$. Then either $f$ is rotationally symmetric, or $M^{n+1} \cong \mathbb{R}^{n+1}$ and $g$ is the flat metric.

Proof. Write $g=d r^{2}+\omega^{2}(r) \tilde{g}$ for $r \in(A, \Omega)$, and fix local coordinates $r=$ $\theta^{0}, \theta^{1}, \ldots, \theta^{n}$ on a neighborhood $(A, \Omega) \times \mathcal{U}$ about any point. Observe that for each fixed $r$, Equation (6) is a tensorial identity on $S^{n}$, and that we may therefore differentiate it using the Levi-Civita connection $\widetilde{\nabla}$ of $\tilde{g}$ to obtain

$$
\widetilde{\nabla}_{k} \widetilde{\nabla}_{i} \widetilde{\nabla}_{j} f+\omega \omega^{\prime} f_{0 k} \tilde{g}_{i j}=0 .
$$

Hence $\widetilde{\nabla}_{k} \widetilde{\nabla}_{i} \widetilde{\nabla}_{j} f-\widetilde{\nabla}_{i} \widetilde{\nabla}_{k} \widetilde{\nabla}_{j} f=\omega \omega^{\prime}\left(f_{0 i} \tilde{g}_{j k}-f_{0 k} \tilde{g}_{i j}\right)$. On the other hand, since $\widetilde{R}_{i j k l}=\tilde{g}_{i l} \tilde{g}_{j k}-\tilde{g}_{i k} \tilde{g}_{j l}$, the standard commutation identities imply

$$
\begin{aligned}
\widetilde{\nabla}_{k} \widetilde{\nabla}_{i} \widetilde{\nabla}_{j} f-\widetilde{\nabla}_{i} \widetilde{\nabla}_{k} \widetilde{\nabla}_{j} f & =-\widetilde{R}_{k i j l} \tilde{g}^{l m} f_{m} \\
& =-\left(\tilde{g}_{i j} \tilde{g}_{k l}-\tilde{g}_{i l} \tilde{g}_{j k}\right) \tilde{g}^{l m} f_{m}=f_{i} \tilde{g}_{j k}-f_{k} \tilde{g}_{i j} .
\end{aligned}
$$

Combining the two, we find $\left(\omega \omega^{\prime} f_{0 i}-f_{i}\right) \tilde{g}_{j k}=\left(\omega \omega^{\prime} f_{0 k}-f_{k}\right) \tilde{g}_{i j}$, and tracing yields $(n-1)\left(\omega \omega^{\prime} f_{0 i}-f_{i}\right)=0$ for all $i=1, \ldots, n$. Together with (5), we conclude

$$
(n-1)|\widetilde{\nabla} f|_{\tilde{g}}^{2}\left[1-\left(\omega^{\prime}\right)^{2}\right]=0 .
$$

Since we assume $n>1$, if $(X f)\left(r_{0}, \theta_{\mathbf{0}}\right) \neq 0$ for some $X \in T_{\left(r_{0}, \theta_{\mathbf{0}}\right)} M^{n+1}$ tangent to the $S^{n}$ factor, we must have $\left|\omega^{\prime}\right| \equiv 1$ and $\omega^{\prime \prime} \equiv 0$ on an interval $(a, b) \subset(A, \Omega)$ containing $r_{0}$. But, by Equation (2), this means that $v_{1}=v_{2}=0$ on $(a, b)$. We claim that $v_{1}=v_{2}=0$ on the entire interval $(A, \Omega)$.

Let $\beta=\sup \left\{r<\Omega \mid\left(\omega^{\prime}\right)^{2}=1\right.$ on $\left.(a, r)\right\}$. If $\beta<\Omega$, by equations (6) and (8), we must have $(\widetilde{\nabla} \widetilde{\nabla} f)(\beta, \cdot)=0, \quad \omega^{\prime}(\beta)=\sigma \in\{ \pm 1\}, \quad \omega^{\prime \prime}(\beta)=0, \omega(\beta)>0$, and $f_{0}(\beta, \cdot)=\sigma \lambda \omega(\beta)$. Moreover, for some small $\epsilon, f$ is a function only of $r$ on $[\beta, \beta+\epsilon)$, and on this interval, $f^{\prime}$ and $\omega$ satisfy the system (7) with the above initial conditions. But one may check that the functions

$$
\bar{\omega}(r)=\sigma(r-\beta)+\omega(\beta) \quad \text { and } \quad \bar{f}^{\prime}(r)=\lambda((r-\beta)+\omega(\beta))
$$

also satisfy (7) and agree with $\omega$ and $f$ at $r=\beta$. Therefore, by uniqueness ${ }^{2}$, these solutions must coincide and it follows that $\left(\omega^{\prime}\right)^{2}=\left(\bar{\omega}^{\prime}\right)^{2}=1$ on the interval $[\beta, \beta+\epsilon)$, contradicting our choice of $\beta$. So $g$ is flat on $(a, \Omega) \times S^{n}$. Using a

${ }^{2}$ Writing $x=\omega^{\prime}$, and $u=f^{\prime}$, we may recast (7) as a first-order system

$$
\begin{aligned}
\omega^{\prime} & =x:=F(\omega, x, u), \\
x^{\prime} & =x u-\lambda \omega+(n-1)\left(1-x^{2}\right) / \omega:=G(\omega, x, u), \\
u^{\prime} & =n x u / \omega+(n-1)\left(n\left(1-x^{2}\right) / \omega^{2}-\lambda\right):=H(\omega, x, u) .
\end{aligned}
$$

Since $F, G, H$ are $C^{\infty}$ on the region $\{\omega \neq 0\}$, the asserted uniqueness follows from standard ODE theory. 
similar argument at the other endpoint $a$, can show that $g$ must be flat on the entire cylinder $(A, \Omega) \times S^{n}$. But this means either $\omega^{\prime} \equiv 1$ or $\omega^{\prime} \equiv-1$, so $g$ cannot extend to a smooth metric on the sphere $S^{n+1}$ or to a complete metric on the cylinder. The only possibility is $\omega^{\prime} \equiv 1$ and $M^{n+1} \cong \mathbb{R}^{n+1}$.

2.3. An equivalent first order system and its linearization. In view of the result of the last section, we now assume $f=f(r)$. We are interested in solutions $(\omega(r), f(r))$ to the system (7) for which $\omega$ is strictly positive.

As Ivey observes in [Chow et al. 2007], (7) is invariant under translations of $r$ and $f$. By introducing the variables $x=\omega^{\prime}$ and $y=n \omega^{\prime}-\omega f^{\prime}$ which share this invariance and an independent variable $t$ that satisfies $d t=1 / \omega d r$, one obtains the first order system

$$
\begin{aligned}
& d \omega / d t=x \omega, \\
& d x / d t=x^{2}-x y+n-1-\lambda \omega^{2}, \\
& d y / d t=x y-n x^{2}-\lambda \omega^{2} .
\end{aligned}
$$

Any solution to (7) gives rise to a trajectory of (9) and conversely, from a trajectory $(\omega(t), x(t), y(t))$ of (9), one may recover $r, \omega(r)$, and $f(r)$ by a succession of quadratures; see [Ivey 1992]. Consequently, it suffices to analyze solutions to the simpler system (9). We take $(\omega, x, y)$ as coordinates on the phase space $\mathbb{R}^{3}$ and restrict our attention to trajectories lying in the half space $\omega>0$.

For $n>1$, system (9) has the two equilibrium points $P_{0}:=(0,1, n)$ and $P_{1}:=$ $(0,-1,-n)$. Denoting the right hand side of (9) by $\Phi$, one finds

$$
d \Phi_{P_{0}}=-d \Phi_{P_{1}}=\left(\begin{array}{ccr}
1 & 0 & 0 \\
0 & 2-n & -1 \\
0 & -n & 1
\end{array}\right),
$$

which has eigenvalues 2,1 , and $1-n$. Since we assume $n \geq 2$, both $P_{0}$ and $P_{1}$ are saddle points: $P_{0}\left(P_{1}\right)$ lying at the intersection of a two-dimensional unstable (stable) manifold and a one-dimensional stable (unstable) manifold. In particular, there is a one-parameter family of trajectories in the half-space $\omega>0$ initially tangent to $(1,0,0)$, among which, in light of Lemma 2, lie the trajectories which give rise to smooth solutions on $S^{n+1}$ and $\mathbb{R}^{n+1}$ (see Examples 5 and 6 below). Trajectories which correspond to smooth solutions on $S^{n+1}$ must, in addition, tend to $P_{1}$ as $t \rightarrow \infty$ and hence lie in the intersection of the global unstable and stable manifolds of $P_{0}$ and $P_{1}$, respectively.

Remark 4. If $L: \mathbb{R}^{3} \rightarrow \mathbb{R}^{3}$ is the map $(\omega, x, y) \mapsto(\omega,-x,-y)$, then from any solution $\gamma(t)=(\omega(t), x(t), y(t))$ of (9) on $(S, T)$, one may obtain a new solution

$$
\bar{\gamma}(t):=L(\gamma(\tau(t)))=(\omega(\tau(t)),-x(\tau(t)),-y(\tau(t)))
$$


on an appropriate interval $(\bar{S}, \bar{T})$, where $\tau$ is chosen to satisfy $d \tau / d t=-1$ and the conditions $\tau(\bar{S})=T$ and $\tau(\bar{T})=S$.

By use of this device, one immediately finds that the global stable and unstable manifolds of $P_{i}, S_{i}$ and $U_{i}$ for $i=1,2$ are related by $L\left(S_{0}\right)=U_{1}$ and $L\left(U_{0}\right)=S_{1}$. Moreover, if a set $V \subset \mathbb{R}^{3}$ is preserved by the system (9) for increasing $t$ (that is, $\gamma\left(t_{0}\right) \in V$ implies $\gamma(t) \in V$ for $t>t_{0}$ as long as the solution is defined), then $L(V)$ is preserved by the system for decreasing $t$.

2.4. The standard examples. It is worthwhile to recall the standard solutions to (7) and locate the corresponding solution to (9) in $\omega x y$-space. Theorem 1 says that this list essentially exhausts the possibilities for complete solutions.

Example 5 (Round sphere). The condition $f \equiv$ const in (7) leads to the constant curvature soliton structure

$$
\omega(r)=\sqrt{n / \lambda} \sin (\sqrt{\lambda / n} r) \text { and } f(r)=\mathrm{const}
$$

on the sphere $S^{n+1}$. The corresponding trajectory in $\omega x y$-space is the elliptical arc $\left\{n x^{2}+\lambda \omega^{2}=n\right\}$ lying in the plane $\{y=n x\}$ joining $P_{0}$ and $P_{1}$.

Example 6 (Gaussian soliton). The condition $\omega^{\prime} \equiv 1$ in (7) leads to the flat soliton structure

$$
\omega(r)=r \quad \text { and } \quad f(r)=\lambda r^{2} / 2+\text { linear }
$$

on $\mathbb{R}^{n+1}$ and corresponds to the trajectory $y=n-\omega^{2}$ in the plane $\{x=1\}$ emanating from $P_{0}$. Applying the device of Remark 4, one obtains a similar trajectory in the plane $\{x=-1\}$, which satisfies $y=-n+\lambda \omega^{2}$ and tends to $P_{1}$ as $t \rightarrow \infty$.

Example 7 (Standard cylinder). The condition $\omega^{\prime} \equiv 0$ in (7) leads to the structure

$$
\omega(r)=\sqrt{(n-1) / \lambda} \text { and } f(r)=\lambda r^{2} / 2+\text { linear }
$$

on $\mathbb{R} \times S^{n}$ corresponding to the line $\{(\sqrt{(n-1) / \lambda}, 0, y)\}$ in $\omega x y$-space.

Remark 8. The flat and cylindrical solutions described in the last two examples are the only ones for which $\omega^{\prime} \equiv$ const, and the corresponding trajectories in $\omega x y$-space describe the intersections of the planes $\{x=1\},\{x=0\}$, and $\{x=-1\}$ with the set $\left\{x^{2}-x y+n-1-\lambda \omega^{2}=0\right\}$. This leads to an observation we shall use repeatedly: the only solutions $\gamma(t)$ of (9) for which $(d x / d t)\left(t_{0}\right)=0$ and $x\left(t_{0}\right)=1,0$, or -1 at some $t_{0}$ are, by uniqueness, those for which $x(t) \equiv 1,0$, or -1 , respectively.

Finally, we note that by taking $\lambda=0$ in the $x$ and $y$-components of (9) one recovers the analogous system for rotationally symmetric solutions to the steady soliton equation. Thus the trajectories of (9) that lie in the plane $\{\omega=0\}$ are naturally associated with steady soliton structures (although, of course, the warping function $\omega(r)$ of these structures no longer corresponds directly to the $\omega$-coordinate). In 
particular, the (one-dimensional) intersection of the unstable manifold of $P_{0}$ with the plane $\{\omega=0\}$ contains two candidates for a smooth steady soliton on $\mathbb{R}^{n+1}$ : one with negative sectional curvature near the origin, which turns out to be incomplete, and one with positive curvature near the origin, which is the well-known Bryant soliton - a complete steady soliton on $\mathbb{R}^{n+1}$ of strictly positive curvature.

\section{Proof of Theorem 1}

In what follows, $\gamma(t)=(\omega(t), x(t), y(t))$ will represent a trajectory of (9) defined for $t$ in what we may take to be a maximal interval $(S, T)$ with $-\infty \leq S<T \leq \infty$. To reduce the clutter of our expressions, we shall usually suppress the $t$ dependence of the components of the trajectory.

3.1. Some invariant sets. We begin our analysis of the trajectories by observing that the second and third derivatives of $x(t)$ have convenient expressions:

Lemma 9. The $x$-component of any trajectory $\gamma(t)$ of $(9)$ satisfies

$$
\begin{aligned}
& \frac{d^{2} x}{d t^{2}}=(n-1) x\left(x^{2}-1\right)+(3 x-y) \frac{d x}{d t} \\
& \frac{d^{3} x}{d t^{3}}=2 x[(2 n-1) x-y] \frac{d x}{d t}+2\left(\frac{d x}{d t}\right)^{2}+(3 x-y) \frac{d^{2} x}{d t^{2}} .
\end{aligned}
$$

Proof. We compute

$$
\frac{d^{2} x}{d t^{2}}=(2 x-y) \frac{d x}{d t}-x^{2} y+n x^{3}-\lambda \omega^{2} x=(n-1) x\left(x^{2}-1\right)+(3 x-y) \frac{d x}{d t}
$$

and

$$
\begin{aligned}
\frac{d^{3} x}{d t^{3}} & =\left((n-1)\left(3 x^{2}-1\right)+\frac{d x}{d t}-\frac{d y}{d t}\right) \frac{d x}{d t}+2\left(\frac{d x}{d t}\right)^{2}+(3 x-y) \frac{d^{2} x}{d t^{2}} \\
& =2 x((2 n-1) x-y) \frac{d x}{d t}+2\left(\frac{d x}{d t}\right)^{2}+(3 x-y) \frac{d^{2} x}{d t^{2}} .
\end{aligned}
$$

With the above expressions we may easily establish the following qualitative results on the behavior of trajectories of the system.

Lemma 10. The regions

$$
\{x \geq 1, d x / d t \geq 0\}, \quad\{x \leq-1, d x / d t \leq 0\}, \quad\{y \leq 0\}
$$

are preserved under system (9) for increasing $t$, and

$$
\{x \geq 1, d x / d t \leq 0\}, \quad\{x \leq-1, d x / d t \geq 0\}, \quad\{y \geq 0\}
$$

are preserved for decreasing $t$. 
Proof. By (11), if $(d x / d t)\left(t_{0}\right)=0$ at some $t_{0}$ with $x\left(t_{0}\right)>1$, then $\left(d^{2} x / d t^{2}\right)\left(t_{0}\right)>0$, and hence both $d x / d t$ and $x$ continue to increase. By Remark 8, we cannot have $d x / d t=0$ and $x=1$ simultaneously unless $x \equiv 1$. The argument for the case $x \leq-1$ and $d x / d t \leq 0$ is similar.

To see that $\{y \leq 0\}$ is preserved, observe that $d y / d t=-n x^{2}-\lambda \omega^{2}<0$ whenever $y=0$.

The preservation of the remaining sets for decreasing $t$ follows by applying previous results to the trajectory $\bar{\gamma}(t)=L(\gamma(\tau))$ constructed as in Remark 4 .

Lemma 11. (1) If there exists $t_{0} \in(S, T)$ at which $x\left(t_{0}\right)=0, y\left(t_{0}\right) \leq 0$, and $(d x / d t)\left(t_{0}\right)>0$, then $x(t)$ (and $\left.d x / d t\right)$ increases until $\gamma(t)$ enters the region $\{x>1\}$.

(2) If there exists $t_{0} \in(S, T)$ at which $x\left(t_{0}\right)=0, y\left(t_{0}\right) \geq 0$, and $(d x / d t)\left(t_{0}\right)>0$, then $x(t)<0$ and $(d x / d t)(t)>0$ for all $t<t_{0}$ and there exists $a t_{1} \leq t_{0}$ such that $x(t)<-1$ for all $t<t_{1}$.

In particular, in view of Lemma 10, if the trajectory $\gamma$ enters the region $\{x<0\}$, either it remains there or eventually lies in the region $\{x>1\}$.

Proof. In case (1), we have $y(t)<0$ for all $t>t_{0}$ by Lemma 10, and we have $\left(d^{2} x / d t^{2}\right)\left(t_{0}\right) \geq 0$ by Equation (11). Since by (12) $\left(d^{3} x / d t^{3}\right)>0$ on the region

$$
\left\{x \geq 0, \quad d x / d t>0, \quad d^{2} x / d t^{2} \geq 0\right\},
$$

we have $\left(d^{2} x / d t^{2}\right)(t)>0$ for all $t>t_{0}$. Consequently, $x(t)>(d x / d t)\left(t_{0}\right)\left(t-t_{0}\right)$. As bounds on $x$ imply bounds on the derivatives of $\omega$ and $y$, the solution cannot expire while $0<x<1$. Since the interval $(S, T)$ is maximal, $x>1$ eventually.

Case (2) then follows from case (1) by considering again the trajectory $\bar{\gamma}(t)$ constructed according to the device in Remark 4: If $x\left(t_{0}\right)=0, y\left(t_{0}\right) \geq 0$, and $\frac{d x}{d t}\left(t_{0}\right)>0$, then $\bar{x}\left(t_{0}\right)=0, \bar{y}\left(t_{0}\right) \leq 0$, and $(d \bar{x} / d t)\left(t_{0}\right)>0$, and the corresponding interval of definition $(\bar{S}, \bar{T})$, satisfying $\tau(\bar{T})=S$ and $\tau(\bar{S})=T$, will also be maximal.

\subsection{Proof of the case $M^{n+1} \cong S^{n+1}$.}

Theorem 12. Suppose $\left(S^{n+1}, g\right)$ is a rotationally symmetric shrinking soliton. Then $g$ has positive curvature operator.

Proof. Recall from Section 2.1 that, for a rotationally symmetric metric, the positivity of the curvature operator is implied by that of the sectional curvatures $v_{1}$ and $v_{2}$ which, in the $(\omega, x, y)$-coordinates, have the expressions

$$
v_{1}=-\frac{1}{\omega^{2}} \frac{d x}{d t} \quad \text { and } \quad v_{2}=\frac{\left(1-x^{2}\right)}{\omega^{2}} .
$$

Any trajectory $\gamma(t)$ of system (9) that corresponds to a smooth soliton structure on the sphere must tend to $P_{0}=(0,1, n)$ as $t \rightarrow-\infty$ and to $P_{1}=(0,-1,-n)$ as 
$t \rightarrow \infty$. Thus, by Lemma 10, we must have $-1<x(t)<1$ (hence $v_{2}(t)>0$ ) for all $t$ and $d x / d t<0$, at least initially. We wish to show $d x / d t<0$ for all $t$.

By Equation (11) of Lemma 9, $d^{2} x / d t^{2}<0$ at critical points of $x$ in the region $\{0<x<1\}$, so $d x / d t$ remains strictly negative on this region and, by Remark 8 , cannot vanish on $\{x=0\}$. Since $\gamma(t)$ must tend to $P_{1}$ as $t \rightarrow \infty$, it must, in particular, enter the region $\{x<0\}$, and, in view of Lemma 11, remain there for all subsequent $t$. Since $d^{2} x / d t^{2}>0$ at critical points of $x$ in the region $\{-1<x<0\}$, $d x / d t$ must therefore remain strictly negative if $x$ is to approach -1 . So $d x / d t<0$ always, and thus for any trajectory emanating from $P_{0}$ and tending to $P_{1}$, we have $v_{1}>0$ and $v_{2}>0$ for all $t$ as claimed.

Taking limits, one finds that at the "poles" $r=A$ and $r=\Omega$, the sectional curvatures agree and are at least nonnegative. One may therefore apply [Hamilton 1986, Lemma 8.2] to conclude that the curvature operator $\operatorname{Rm}(g): \wedge^{2} \rightarrow \wedge^{2}$ is of constant rank and therefore strictly positive everywhere.

That $g$ has constant sectional curvature then follows immediately from this:

Theorem [Böhm and Wilking 2006, Theorem 1]. On a compact manifold, the normalized Ricci flow evolves a Riemannian metric with a 2-positive curvature operator to a limit metric with constant sectional curvature.

That $f \equiv$ const in case (1) of Theorem 1 then follows by substituting $v_{1}=v_{2}=$ const into (7) or, alternatively, by considering the identity $R+|\nabla f|^{2}-2 \lambda f=$ const, which is valid on any gradient Ricci shrinking soliton; see for example [Chow et al. 2007]. If $f$ attains its maximum and minimum at the points $x_{M}$ and $x_{m}$, respectively, then the identity implies $f\left(x_{M}\right)=f\left(x_{m}\right)$ since $R$ is constant.

\subsection{The asymptotic behavior of trajectories corresponding to complete, non-}

compact metrics. Hereafter, we shall consider solutions $\gamma(t)$ satisfying one or both of the conditions

$$
\int_{t_{0}}^{T} \omega(\sigma) d \sigma=\infty=\lim _{t \rightarrow T} r(t)
$$

and

$$
\int_{S}^{t_{0}} \omega(\sigma) d \sigma=\infty=-\lim _{t \rightarrow S} r(t)
$$

for any $t_{0} \in(S, T)$. Condition (13) is necessarily satisfied by any trajectory corresponding to a complete metric on $\mathbb{R}^{n+1}$, and both (13) and (14) are necessarily satisfied by any trajectory corresponding to a complete metric on $\mathbb{R} \times S^{n}$. As we shall see, these conditions impose rather stringent conditions on the asymptotic behavior of a trajectory.

We remark that if $\gamma(t)$ satisfies (13) then $\bar{\gamma}(t)=L(\gamma(\tau))$ satisfies (14) and vice versa. Thus, from the following results, which apply to trajectories satisfying the 
"forward" condition (13), we may easily obtain corresponding results for trajectories satisfying the "backward" condition (14). These results will be collected in Lemma 17, below.

We begin with a simple consequence of the forwards extendability condition by which we may eventually obtain knowledge of the sign of the $y$-component.

Lemma 13. Along any trajectory $\gamma(t)$, the quantity $Q=y / \omega$ is strictly decreasing. If $\gamma(t)$ satisfies (13), then $\lim _{t \rightarrow T} Q=-\infty$. In particular, $y$ eventually becomes negative.

Proof. We compute $d Q / d t=-n x^{2} / \omega-\lambda \omega<0$. Integrating, we find that, for any $S<t_{0}<t<T$,

$$
\frac{y}{\omega}(t) \leq \frac{y}{\omega}\left(t_{0}\right)-\lambda \int_{t_{0}}^{t} \omega(\sigma) d \sigma .
$$

The following observation is also immediate.

Lemma 14. If $\gamma(t)$ satisfies (13), then $\lim \sup _{t \rightarrow T} x(t) \geq 0$.

Proof. If $x(t)<-\delta$ on some $(a, T) \subset(S, T)$, then $\omega(t) \leq C e^{-\delta t}$ on the same interval, and $\gamma(t)$ cannot satisfy (13).

Thus, in view of Lemma 11, no trajectory satisfying (13) can enter the region $\{x<-1\}$. That no trajectory satisfying (13) can enter the region $\{x>1\}$ is true (as we prove next) but less obvious since $\omega(t) \rightarrow \infty$. Taken together, Lemmas 11, 14 , and 15 prove that any complete metric on $\mathbb{R}^{n+1}$ satisfies $v_{2} \geq 0$.

The proof follows the lines of an argument due to Bryant and Ivey (see [Chow et al. 2007]) demonstrating the incompleteness of a similar trajectory of the steady soliton system. In fact, if one regards the trajectories of (9) in the plane $\{\omega=0\}$ as trajectories of the analogous system for steady solitons (see the remarks at the end of Section 2.4), then Ivey's argument pertains to the trajectory in the plane emerging from $P_{0}$ in the direction opposite the Bryant soliton. The following lemma then may be viewed as extending his finding to the neighboring family of trajectories in the unstable manifold $U_{0}$ which populate the sector between the flat trajectory with $x \equiv 0$ and the plane $\{\omega=0\}$. These trajectories correspond to metrics of strictly negative sectional curvature and are all incomplete. However, Ivey's argument does not carry over directly, because, in the expression for $d x / d t$ in the shrinking case, one has to contend with an additional term $\left(-\lambda \omega^{2}\right)$ of uncooperative sign.

Lemma 15. Suppose $x\left(t_{0}\right)>1$ and $(d x / d t)\left(t_{0}\right)>0$ at some $t_{0} \in(S, T)$. Then $\int_{t_{0}}^{T} \omega(\sigma) d \sigma<\infty$.

Proof. We proceed by contradiction. Suppose $\gamma(t)$ satisfies (13). Then, by (11), $x(t)>1$ and $(d x / d t)(t)>0$ for $t>t_{0}$, and, by Lemma 13, there is a $t_{1} \in\left(t_{0}, T\right)$ such that $y(t)<0$ for all $t \geq t_{1}$. 
Hence, by (11), we have

$$
\frac{d^{2} x}{d t^{2}} \geq(n-1) x\left(x^{2}-1\right)+3 x \frac{d x}{d t}>\frac{3}{2} \frac{d\left(x^{2}\right)}{d t} .
$$

Now, since the interval $(S, T)$ is assumed maximal, and since bounds on $x$ imply bounds on the derivatives of $y$ and $\omega$, if $T<\infty$ we must have $\lim \sup _{t \rightarrow T}|x(t)|=$ $\lim _{t \rightarrow T} x=\infty$. On the other hand, $x(t)$ is uniformly convex by (15), so even if $T=\infty$ we still have $\lim _{t \rightarrow T} x(t)=\infty$. Returning to (15) with this fact in hand, we find

$$
\frac{d x}{d t} \geq \frac{5}{4} x^{2}+1
$$

for all $t$ greater than some $t_{2} \geq t_{1}$. (The coefficient $5 / 4$ is chosen for convenience and could be replaced by $3 / 2-\epsilon$ for any $\epsilon>0-$ below, we merely require it to be greater than one.) From this equation it follows that $T<\infty$ and

$$
\frac{d}{d t} \arctan \left(\frac{x \sqrt{5}}{2}\right) \geq \frac{\sqrt{5}}{2}
$$

which implies $x(t) \leq 4 /(5(T-t))$ for $t$ sufficiently close to $T$. Now, since $d \log \omega / d t=x$, integrating and applying this bound yields $\omega(t) \leq C_{2} /(T-t)^{4 / 5}$ for some constant $C_{2}$, contradicting (13).

Together, Lemmas 10 and 15 allow us to restrict our attention to trajectories that remain in the region $\{-1<x<1\}$, and, consequently, to those with infinite existence time $t \in(S, \infty)$, since a trajectory with bounded $x$ cannot satisfy condition (13) on a interval bounded above. Along such trajectories, $y$ becomes negative and Lemma 11 implies that eventually $x$ acquires a constant sign. As a consequence, we obtain the following refinement of Lemma 13:

Lemma 16. If $\gamma(t)$ is a trajectory of $(9)$ defined on $(S, \infty)$ satisfying condition (13) and $-1<x(t)<1$, then $\lim _{t \rightarrow \infty} y(t)=-\infty$.

Proof. Suppose $\lim \sup _{t \rightarrow \infty} y(t) \geq-M$ for some $M>1$, and choose $t_{k} \in(S, \infty)$ such that $t_{k} \nearrow \infty$ and $y\left(t_{k}\right) \geq-M$. Then, by Lemma $13, \lim _{k \rightarrow \infty} \omega\left(t_{k}\right)=0$. Since $x$ eventually acquires a constant sign, and $d \omega / d t=x \omega$, we must have $x(t) \leq 0$ eventually, and $\omega(t)$ must tend to 0 outright as $t \rightarrow \infty$.

Now, Equation (11) shows that $x(t)$ cannot attain a local maximum on the range $\{-1<x \leq 0\}$ unless $x=0$, and we know $x=0$ and $d x / d t=0$ simultaneously only if $x \equiv 0$, in which case $d y=-(n-1) d t$. Otherwise, $x$ is eventually monotonic in $t$ and either increases or decreases to a limit $\bar{x} \in[-1,0]$. By the remarks preceding this lemma, we cannot have $\bar{x}<0$ if the trajectory is to satisfy condition (13). So assume $\bar{x}=0$, which implies $d x / d t>0$ eventually. Then, for any $\epsilon$, we can choose $t_{\epsilon}$ such that $t>t_{\epsilon}$ implies both $y(t)<0$ and $n x^{2}+\lambda \omega^{2}<\epsilon$. For such $t$, we have $d y / d t=x y-n x^{2}-\lambda \omega^{2}>-\epsilon$. 
Fixing $\epsilon<(n-1) / 2$, we find, each for $k$, that $y(t)>-M-\epsilon\left(t-t_{k}\right)$ and

$$
\frac{d x}{d t}(t)=x^{2}-x y+n-1-\lambda \omega^{2}>\frac{n-1}{2}+x\left(t_{k}\right)\left(M+\left(\epsilon\left(t-t_{k}\right)\right),\right.
$$

where, in the last inequality, we used that $x$ is monotonically increasing.

Hence

$$
x(t)-x\left(t_{k}\right)>\left(\frac{n-1}{2}+M x\left(t_{k}\right)\right)\left(t-t_{k}\right)+\epsilon \frac{x\left(t_{k}\right)}{2}\left(t-t_{k}\right)^{2} .
$$

For $k>>0, M x\left(t_{k}\right)>-(n-1) / 4$, so that the above (with the monotonicity of $x(t))$ implies that there exists $\delta=\delta(M, \epsilon, n)>0$ and a subsequence $t_{k_{j}} \rightarrow \infty$ such that $x\left(t_{k_{j+1}}\right)>x\left(t_{k_{j}}\right)+\delta$ for all $j$. This contradicts that $x \nearrow 0$ and proves $\lim \sup _{t \rightarrow \infty} y(t)=-\infty$.

3.4. Proof of the case $M^{n+1} \cong \mathbb{R}^{n+1}$. We use the results of the last section.

Proof of claim (2) of Theorem 1. Since the underlying manifold $M^{n+1}$ is diffeomorphic to $\mathbb{R}^{n+1}$, the smooth extension of the metric to the origin $r=0$ then requires that $S=-\infty$ and that our solution $\gamma(t)=(\omega(t), y(t), \omega(t))$ of $(9)$ satisfies $\lim _{t \rightarrow-\infty} \gamma(t)=P_{0}=(0,1, n)$.

We claim first that if our trajectory is to satisfy condition (13), then $x \leq 1$. For if ever $x>1$, since $\lim _{t \rightarrow-\infty} x(t)=1$, we would have to have $(d x / d t)\left(t_{0}\right)>0$ and $x\left(t_{0}\right)>1$ at some earlier $t_{0}$. But then, by Lemmas 10 and 15, the $x$-component would blow up too fast for $\gamma(t)$ to satisfy (13). So we must have $x \leq 1$ for all $t$.

Then, if ever $x=1$, we must also have $d x / d t=0$ at the same time, which, as pointed out in Remark 8, happens only if $x \equiv 1$ - that is, only if $\gamma(t)$ corresponds to the flat solution of Example 6. We claim that this is the only trajectory emanating from $P_{0}$ that satisfies (13).

We may now assume that $x(t)<1$ on our trajectory and that for some $t_{0}$ (hence all $\left.t<t_{0}\right)$, we have $(d x / d t)\left(t_{0}\right)<0$ and $0<x\left(t_{0}\right)<1$. Since, by Equation (11), $d^{2} x / d t^{2}$ is strictly negative at all critical points of $x$ in the region $0<x<1$, there are two possibilities for our trajectory: either

(1) $x$ decreases monotonically to a limit $\bar{x} \in[0,1)$ as $t \rightarrow \infty$, or

(2) $\gamma(t)$ enters the region $\{x \leq 0\}$ at some time $t=t_{1}$.

Knowing that $y \rightarrow-\infty$ as $t \rightarrow \infty$ (in fact, just knowing that eventually $y<0$ suffices), we can dispose of case (1) by observing that while $x \in[0,1]$,

$$
\frac{d^{2} x}{d t^{2}} \leq(n-1) x\left(x^{2}-1\right)+\epsilon \frac{d x}{d t}<0
$$

once $y<-\epsilon<0$. Hence $x$ eventually becomes negative.

Now, we also know from Lemma 14 that $x$ cannot tend to a negative limit or become strictly less than -1 if condition (13) is to be satisfied. Since $d^{2} x / d t^{2}$ is 
strictly positive at critical points of $x$ in the region $\{-1<x<0\}$, and since the only trajectories with critical points of $x$ on the boundary of this region are classified in Examples 6 and 7 and neither emanate from $P_{0}$, we face only two alternatives:

(2a) either $\gamma$ enters the region $x>0$ again, or

(2b) $x \nearrow 0$ as $t \rightarrow \infty$.

Alternative (2a) is immediately excluded by Lemmas 11 and 15: no trajectory that emanates from $P_{0}$ can satisfy $x\left(t_{0}\right)=0, d x / d t\left(t_{0}\right)>0$, and $y\left(t_{0}\right) \geq 0$ and no trajectory that satisfies $x\left(t_{0}\right)=0, d x / d t\left(t_{0}\right)>0$, and $y\left(t_{0}\right) \leq 0$ can satisfy (13).

For (2b), we observe that since $y \rightarrow-\infty$ as $t \rightarrow \infty$, we have $3 x-y>\epsilon>0$ eventually, and thus we may obtain the analog of Equation (16) for $t$ sufficiently large

$$
\frac{d^{2} x}{d t^{2}}=(n-1) x\left(x^{2}-1\right)+(3 x-y) \frac{d x}{d t}>\epsilon \frac{d x}{d t}>0,
$$

which is incompatible with $x \nearrow 0$ as $t \rightarrow \infty$.

The trajectory $x \equiv 1$ is therefore the unique trajectory emanating from $P_{0}$ satisfying (13), and the proof of the case $M^{n+1} \cong \mathbb{R}^{n+1}$ is complete.

3.5. Proof of the case $M^{\boldsymbol{n}+1} \cong \mathbb{R} \times \boldsymbol{S}^{\boldsymbol{n}}$. As remarked earlier, the results in Section 3.3 regarding trajectories satisfying the forward extendability condition (13) have natural analogs for trajectories satisfying the backward version (14).

Lemma 17. (1) Suppose $\gamma(t)$ satisfies (14).

(a) $\lim _{t \rightarrow S} Q(t)=\infty$ and $y$ is initially positive.

(b) $\liminf _{t \rightarrow S} x(t) \leq 0$.

(c) If $-1<x(t)<1$ for all $t($ so $-S=T=\infty)$, then $\lim _{t \rightarrow-\infty} y(t)=\infty$.

(2) If $x\left(t_{0}\right)<-1$ and $(d x / d t)\left(t_{0}\right)>0$ at some $t_{0} \in(S, T)$, then

$$
\int_{S}^{t_{0}} \omega(\sigma) d \sigma<\infty
$$

Proof. Let $\bar{\gamma}(t)=L(\gamma(\tau(t)))$ be as in Remark 4. Then

$$
\int_{S}^{t_{0}} \omega(\sigma) d \sigma=\int_{t_{0}}^{\bar{T}} \bar{\omega}(\sigma) d \sigma .
$$

Thus, if $\gamma(t)$ satisfies (14), then $\bar{\gamma}(t)$ satisfies (13) and the claims of part (1) follow by the application of Lemmas 13,14 , and 16 to $\bar{\gamma}(t)$.

For part (2), $\bar{x}\left(t_{0}\right)>1$ and $(d \bar{x} / d t)\left(t_{0}\right)>0$ if $x\left(t_{0}\right)<-1$ and $(d x / d t)\left(t_{0}\right)>0$, so Lemma 15 and Equation (19) yield the inequality (18).

Now we turn to the remainder of the proof of Theorem 1. 
Proof of claim (3) of Theorem 1. We shall show that the only trajectory $\gamma(t)$ satisfying both (13) and (14) is that of Example 7 with $x \equiv 0$ and $\omega \equiv \sqrt{(n-1) / \lambda}$.

By Lemmas 10 and 15 and case (2) of Lemma 17, we can assume $-1 \leq x(t) \leq 1$ and consequently also $S=-\infty$ and $T=\infty$. Since neither of the trajectories with $x \equiv \pm 1$ can satisfy both conditions (13) and (14), we may in fact assume $-1<x(t)<1$.

By Lemma 14 and part (b) of Lemma 17, we know $\liminf _{t \rightarrow-\infty} x(t) \leq 0$ and $\lim \sup _{t \rightarrow \infty} x(t) \geq 0$. Thus since $d^{2} x / d t^{2}$ is strictly positive at critical points of $x$ in the region $\{-1<x<0\}$ and strictly negative at these critical points in the region $\{0<x<1\}$, either $\gamma(t)$ crosses the plane $\{x=0\}$ at some time or its $x$-component maintains a constant sign and tends monotonically to 0 as $t \rightarrow \infty$ or $t \rightarrow-\infty$. We claim that this latter option cannot occur. For, in light of the remarks in the preceding paragraph, the only ways $\gamma(t)$ could potentially satisfy the extendability criteria would be $x<0$ and $x \nearrow 0$ as $t \rightarrow \infty$, or $x>0$ and $x \searrow 0$ as $t \rightarrow-\infty$. But the case $x<0, x \nearrow 0$ was eliminated in the argument for claim (2), and the case $x \searrow 0$ as $t \rightarrow-\infty$ reduces to the previous one by considering the trajectory $\bar{\gamma}(t)$.

Thus, we conclude there must exist a $t_{0}$ such that $x\left(t_{0}\right)=0$. If $(d x / d t)\left(t_{0}\right) \neq$ 0 , then we may assume $(d x / d t)\left(t_{0}\right)>0$, because the argument we gave in the case $M^{n+1} \cong \mathbb{R}^{n+1}$ implies that the only trajectories that satisfy Equation (13) and $(d x / d t)\left(t_{0}\right)<0$ initially lie in the region $\{x<-1\}$ and cannot thus satisfy (14). However, if $(d x / d t)\left(t_{0}\right)>0$, then Lemma 11 implies that either again $\gamma(t)$ lies initially in the region $\{x<-1\}$ or eventually in the region $\{x>1\}$, in which case, by Lemma 15 and part (2) of Lemma 17, $\gamma(t)$ can satisfy at most one of the conditions (13) and (14).

Thus we can only have $(d x / d t)\left(t_{0}\right)=0$, which implies that $\gamma$ coincides with the trajectory $x \equiv 0$ and $\omega \equiv \sqrt{(n-1) / \lambda}$ as claimed.

\section{Acknowledgment}

This paper appeared, in a slightly different form, as part of the author's thesis [Kotschwar 2007] at UC San Diego. He wishes to thank the department there for its hospitality and Professors Bennett Chow and Lei Ni for their support and encouragement. The author is also indebted to Professor Thomas Ivey for his analysis of the problem in the case of expanding and steady solitons which served as a guide for the case treated here.

\section{References}

[Böhm and Wilking 2006] C. Böhm and B. Wilking, "Manifolds with positive curvature are space forms", preprint, 2006. To appear in Ann. of Math. arXiv math/0606187v1

[Bryant $\leq 1992$ ] R. Bryant, unpublished notes on Ricci solitons, most of whose contents appear in [Ivey 1992]. 
[Chen et al. 2006] X. Chen, P. Lu, and G. Tian, "A note on uniformization of Riemann surfaces by Ricci flow”, Proc. Amer. Math. Soc. 134:11 (2006), 3391-3393. MR 2007d:53109 Zbl 1113.53042

[Chow and Knopf 2004] B. Chow and D. Knopf, The Ricci flow: an introduction, Mathematical Surveys and Monographs 110, American Mathematical Society, Providence, RI, 2004. MR 2005e: 53101 Zbl 1086.53085

[Chow et al. 2007] B. Chow, S.-C. Chu, D. Glickenstein, C. Guenther, J. Isenberg, T. Ivey, D. Knopf, P. Lu, F. Luo, and L. Ni, The Ricci flow: techniques and applications, I: Geometric aspects, Mathematical Surveys and Monographs 135, American Mathematical Society, Providence, RI, 2007. MR 2302600 Zbl 05149301

[Feldman et al. 2003] M. Feldman, T. Ilmanen, and D. Knopf, "Rotationally symmetric shrinking and expanding gradient Kähler-Ricci solitons", J. Differential Geom. 65:2 (2003), 169-209. MR 2005e:53102 Zbl 1069.53036

[Hamilton 1986] R. S. Hamilton, "Four-manifolds with positive curvature operator", J. Differential Geom. 24:2 (1986), 153-179. MR 87m:53055 Zbl 0628.53042

[Hamilton 1988] R. S. Hamilton, "The Ricci flow on surfaces", pp. 237-262 in Mathematics and general relativity (Santa Cruz, CA, 1986), edited by J. A. Isenberg, Contemp. Math. 71, Amer. Math. Soc., Providence, RI, 1988. MR 89i:53029 Zbl 0663.53031

[Hamilton 1995] R. S. Hamilton, "The formation of singularities in the Ricci flow", pp. 7-136 in Surveys in differential geometry (Cambridge, MA, 1993), vol. 2, edited by C.-C. Hsiung and S.-T. Yau, Int. Press, Cambridge, MA, 1995. MR 97e:53075 Zbl 0867.53030

[Ivey 1992] T. Ivey, On solitons for the Ricci flow, PhD thesis, Duke University, 1992.

[Koiso 1990] N. Koiso, “On rotationally symmetric Hamilton's equation for Kähler-Einstein metrics”, pp. 327-337 in Recent topics in differential and analytic geometry, edited by T. Ochiai, Adv. Stud. Pure Math. 18, Academic Press, Boston, 1990. MR 93d:53057 Zbl 0739.53052

[Kotschwar 2007] B. Kotschwar, Some results on the qualitative behavior of solutions to the Ricci flow and other geometric evolution equations, $\mathrm{PhD}$ thesis, University of California at San Diego, 2007.

[Ni 2005] L. Ni, “Ancient solutions to Kähler-Ricci flow”, Math. Res. Lett. 12:5-6 (2005), 633-653. MR 2006i:53097 Zbl 1087.53061

[Perelman 2003] G. Perelman, "Ricci flow with surgery on three-manifolds", Preprint, 2003. arXiv math.DG/0303109v1

Received September 1, 2006. Revised January 21, 2008.

\section{BRETT KOTSCHWAR}

DEPARTMENT OF MATHEMATICS

MIT 2-304

77 Massachusetts Avenue

CAMBRIDGE, MA 02139-4307

UNITED STATES

kotschwar@math.mit.edu 